\title{
Supporting Information for: Nanoconfinement and salt synergistically suppress crystallization in polyethylene oxide
}

Zheng Zhang, ${ }^{1}$ Junjun Ding, ${ }^{2}$ Benjamin M. Ocko, ${ }^{3}$ Julien Lhermitte, ${ }^{3}$ Joseph Strzalka, ${ }^{4}$ Chang-Hwan Choi, ${ }^{2}$ Frank T. Fisher, ${ }^{2}$ Kevin G. Yager, ${ }^{1}{ }^{1}$ Charles T. Black* ${ }^{1}$

${ }^{1}$ Center for Functional Nanomaterials, Brookhaven National Laboratory, Upton NY, USA

${ }^{2}$ Department of Mechanical Engineering, Stevens Institute of Technology, Hoboken NJ, USA

${ }^{3}$ National Synchrotron Light Source II, Brookhaven National Laboratory, Upton NY, USA

${ }^{4}$ X-ray Science Division, Argonne National Laboratory, Argonne, Illinois 60439, USA

*kyager@bnl.gov, ctblack@bnl.gov 

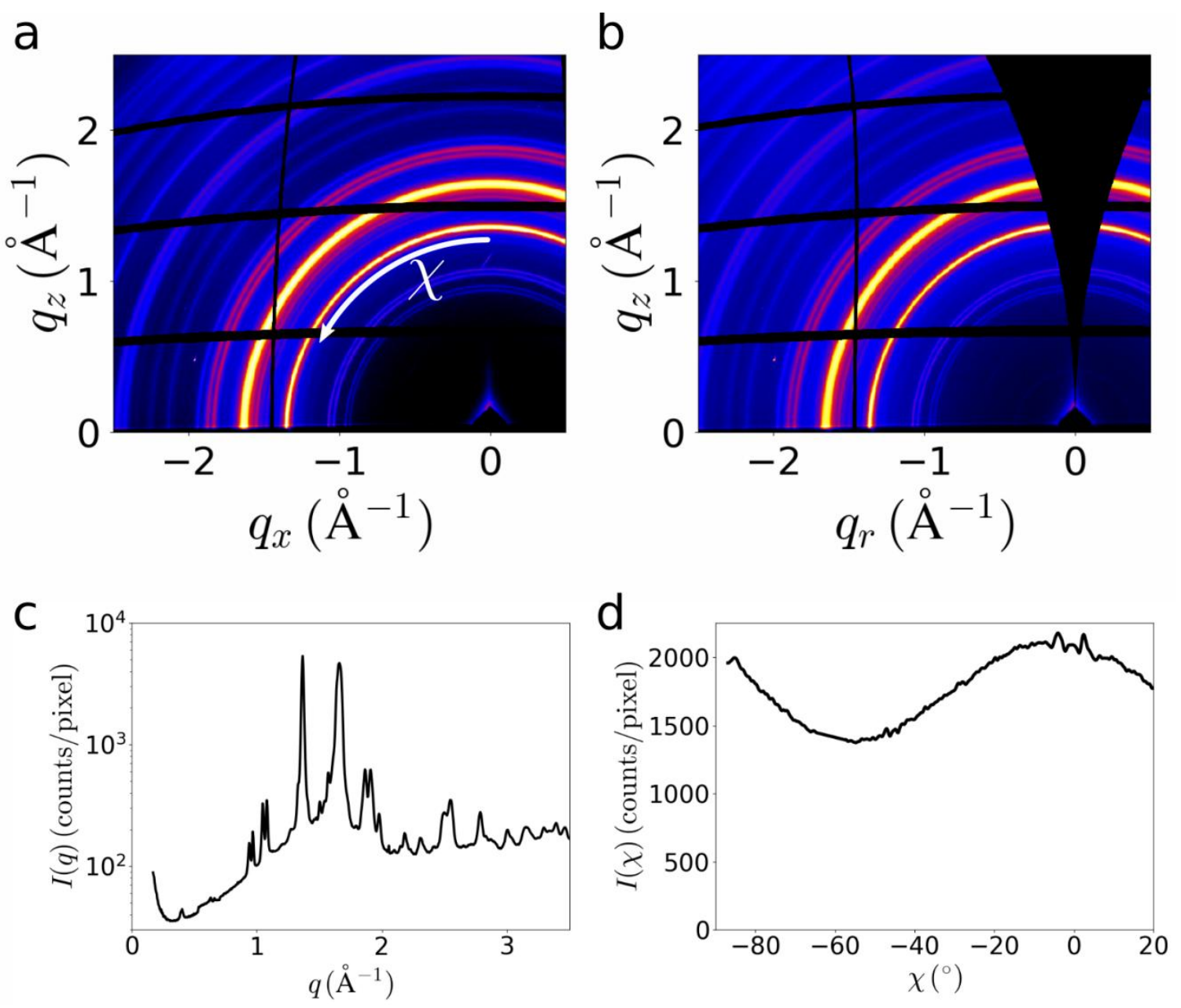

Figure S1: X-ray scattering measurement of a PEO430 $k$ film dropcast from acetonitrile solution. (a) 2D GIWAXS pattern, shown as the $\left(q_{x}, q_{z}\right)$ projection through the three-dimensional reciprocal-space, where the $q_{y}$ component (along the beam direction) is omitted for plotting purposes. (b) The same data, shown using the $\left(q_{r}, q_{z}\right)$ plotting convention, where $q_{r}=\sqrt{q_{x}^{2}+q_{y}^{2}}$ is the in-plane scattering component. (c) Circularly integrated average intensities $I(q)$ as a function $q$. Note that the $q$ is calculated using all scattering components $\left(q_{x}, q_{y}\right.$, and $\left.q_{z}\right)$. (d) Angular cut along an arc of constant $q=1.362 \AA^{-1}$, which corresponds to PEO (120) plane. Intensity variation with respect to $\chi$ is minimal, indicting a roughly isotropic orientation distribution. 


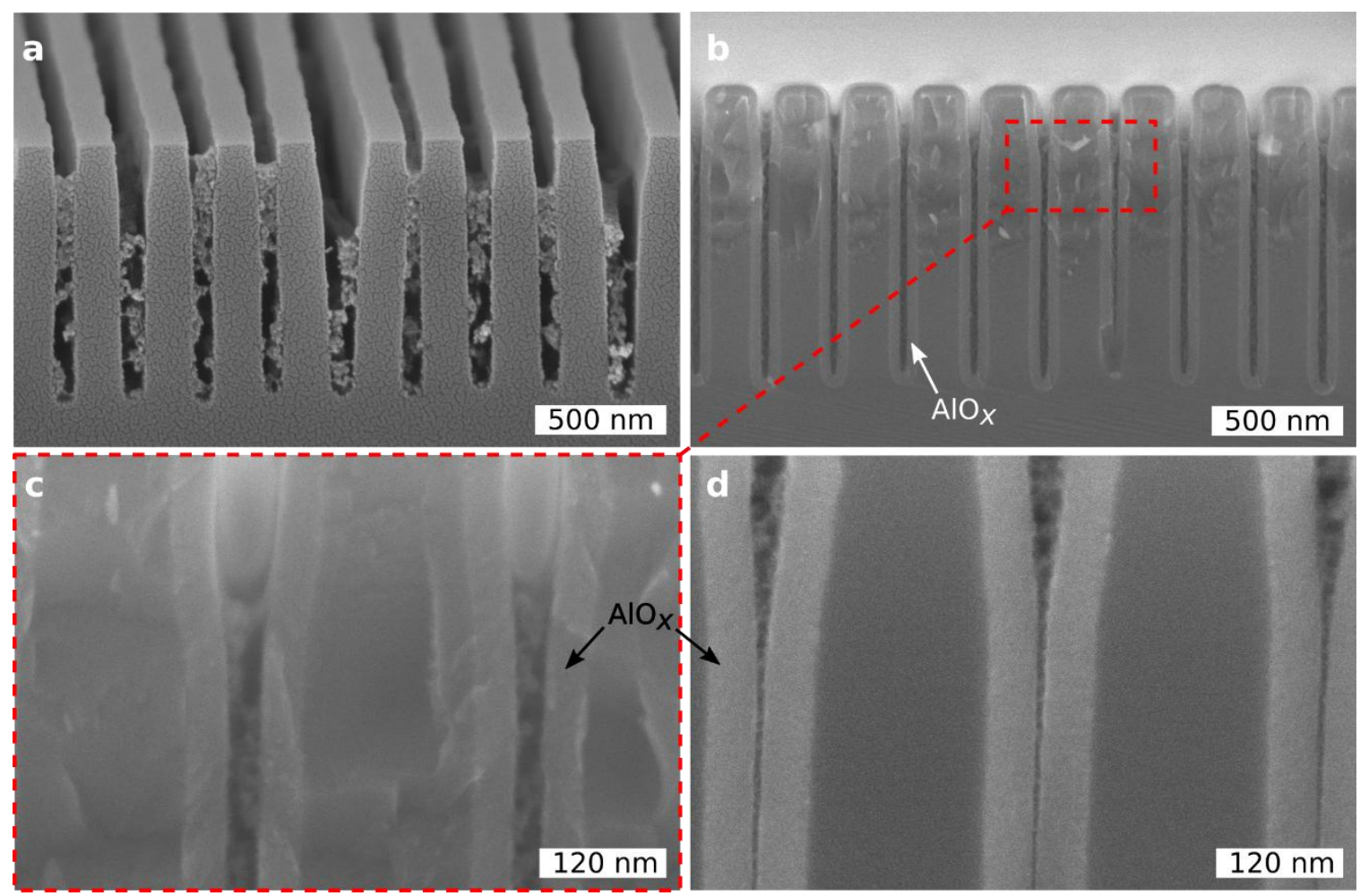

Figure S2: Cross-sectional SEM images of PEO filled in (a) $\sim 110 \mathrm{~nm}$ wide nanogratings; (b-c) $\sim 35 \mathrm{~nm}$ wide nanogratings coated with $\mathrm{AlO}_{x}$; (d) $\sim 8 \mathrm{~nm}$ wide nanogratings coated with $\mathrm{AlO}_{x}$. Note that the 'graininess' of the polymer material is due to the inorganic infiltration used to improve imaging, and that the sub-surface voids arise due to random pull-out of material during cleaving. 

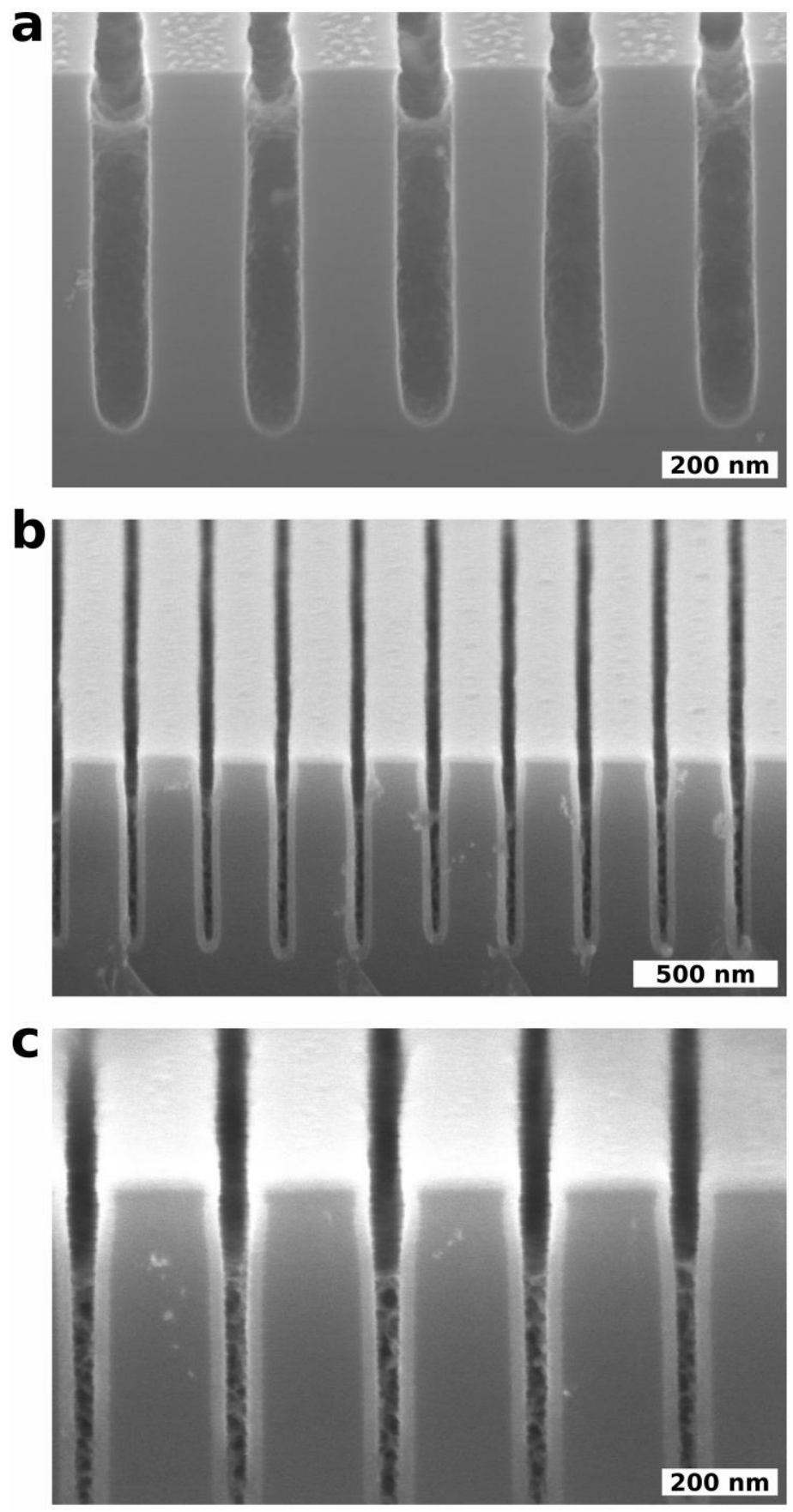

Figure S3: Addition cross-sectional SEM images of PEO filling into nano-gratings. 

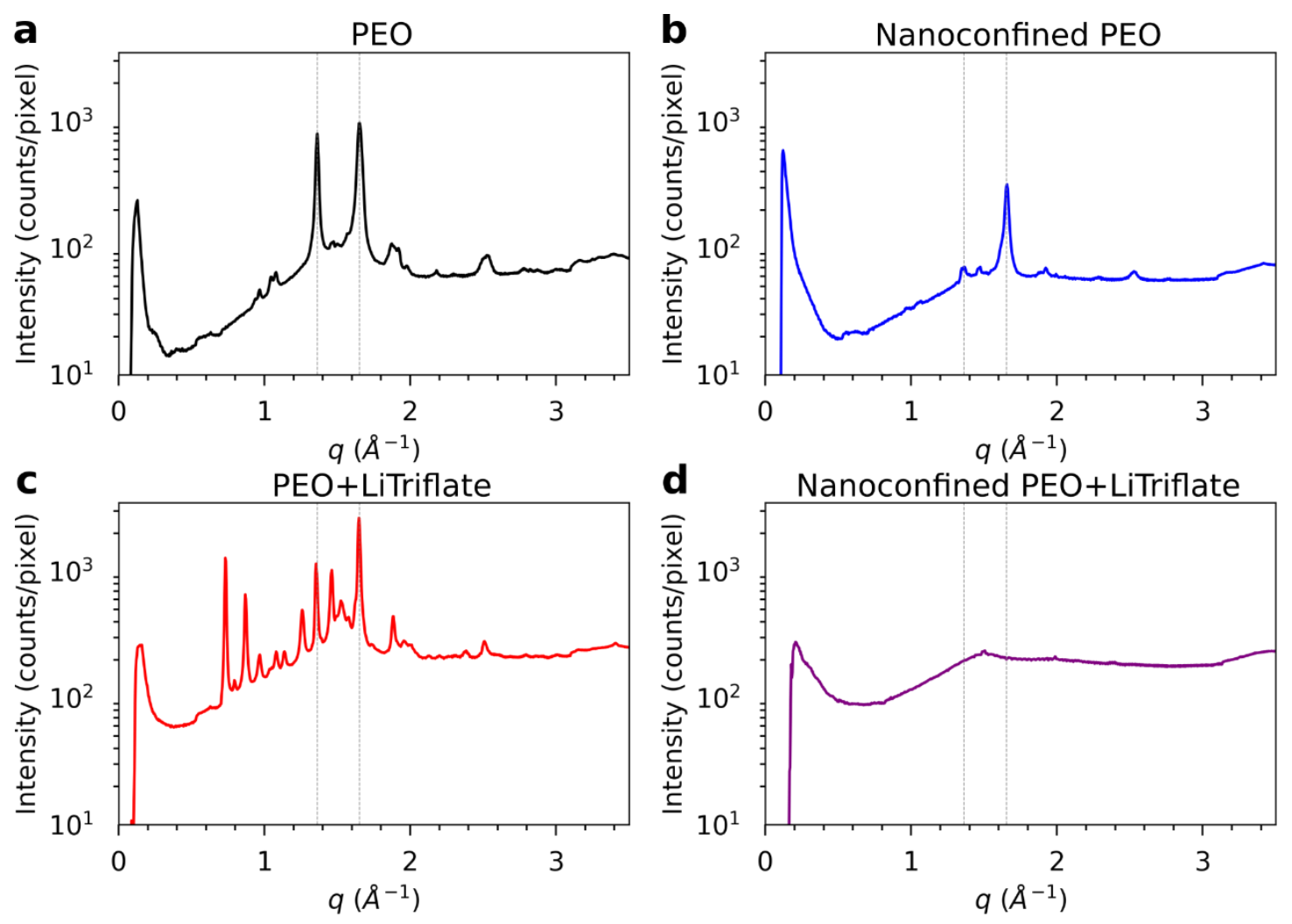

Figure S4: 1D scattering intensity $I(q)$ as a function of $q$ for: (a) spin-coated PEO430 $k$ film; (b) PEO430 $k$ confined in $~ 110 \mathrm{~nm}$ wide nanogratings; (c) spin-coated PEO430k/LiTriflate mixture film; (d) PEO430k/LiTriflate mixture confined in $\sim 110 \mathrm{~nm}$ nanogratings. The combined effect of nanoconfinement and salt strongly suppresses PEO crystallization (as evidenced by the elimination of the strong crystal scattering peaks) and yielding an amorphous material (characterized by a broad amorphous scattering halo instead of the sharp diffraction peaks). We note that some amount of residual crystallinity may still be present, but that the combination of confinement and salt greatly reduce total crystallinity. 

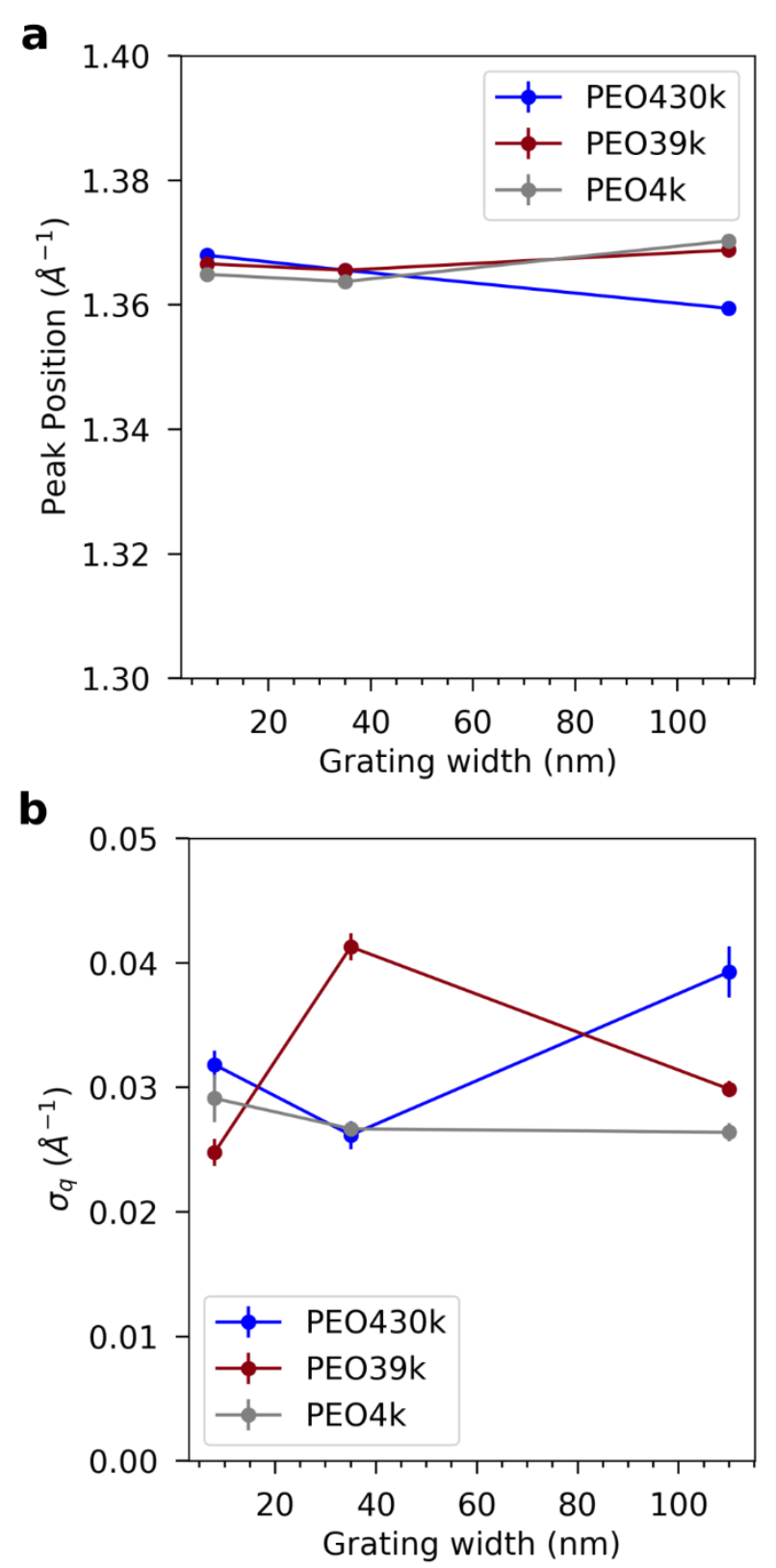

Figure S5: (a) Position and (b) width of the (120) peak of different molecular weights of PEO, as function of nanograting width. The peak position and width are obtained by fitting the 1D circularly averaged intensities $I(q)$ vs. $q$ to a Gaussian function. These parameters do not appreciably change as a function of confinement, indicating that the PEO crystalline unit cell is not perturbed by these confinement conditions. 

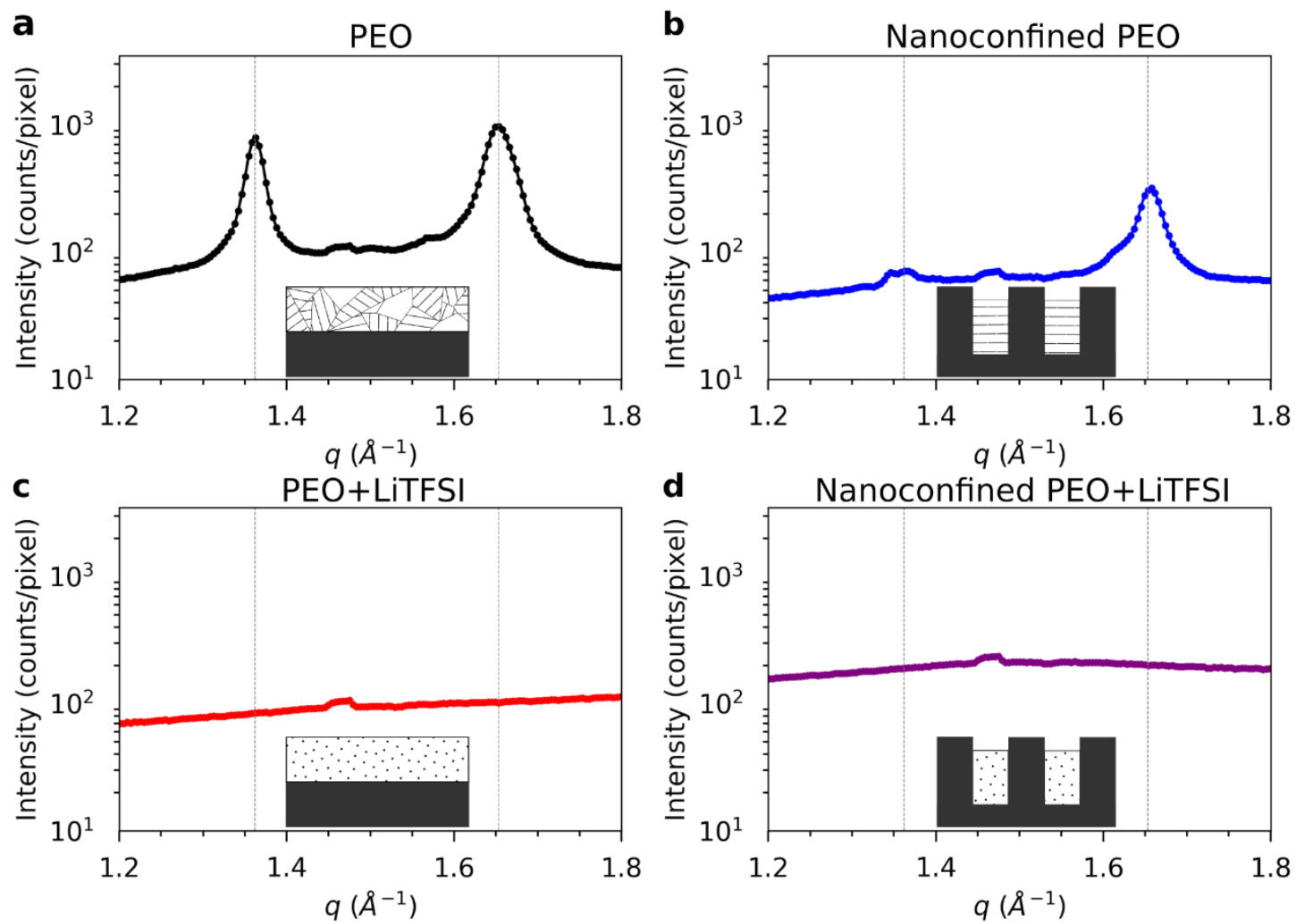

Figure S6: 1D scattering intensity $I(q)$ as a function of $q$ for: (a) spin-coated PEO430k film; (b) PEO430k confined in $\sim 110 \mathrm{~nm}$ wide nanogratings; (c) spin-coated PEO430k/LiTFSI mixture film; (d) PEO430k/LiTFSI mixture confined in $\sim 110 \mathrm{~nm}$ nanogratings. $I(q)$ is calculated by integrating and averaging over $\chi$, for each given $q$. The LiTFSI salt (unlike LiTriflate) disrupts PEO crystallization by itself. When this salt is combined with nanoconfinement, crystallinity remains suppressed. 

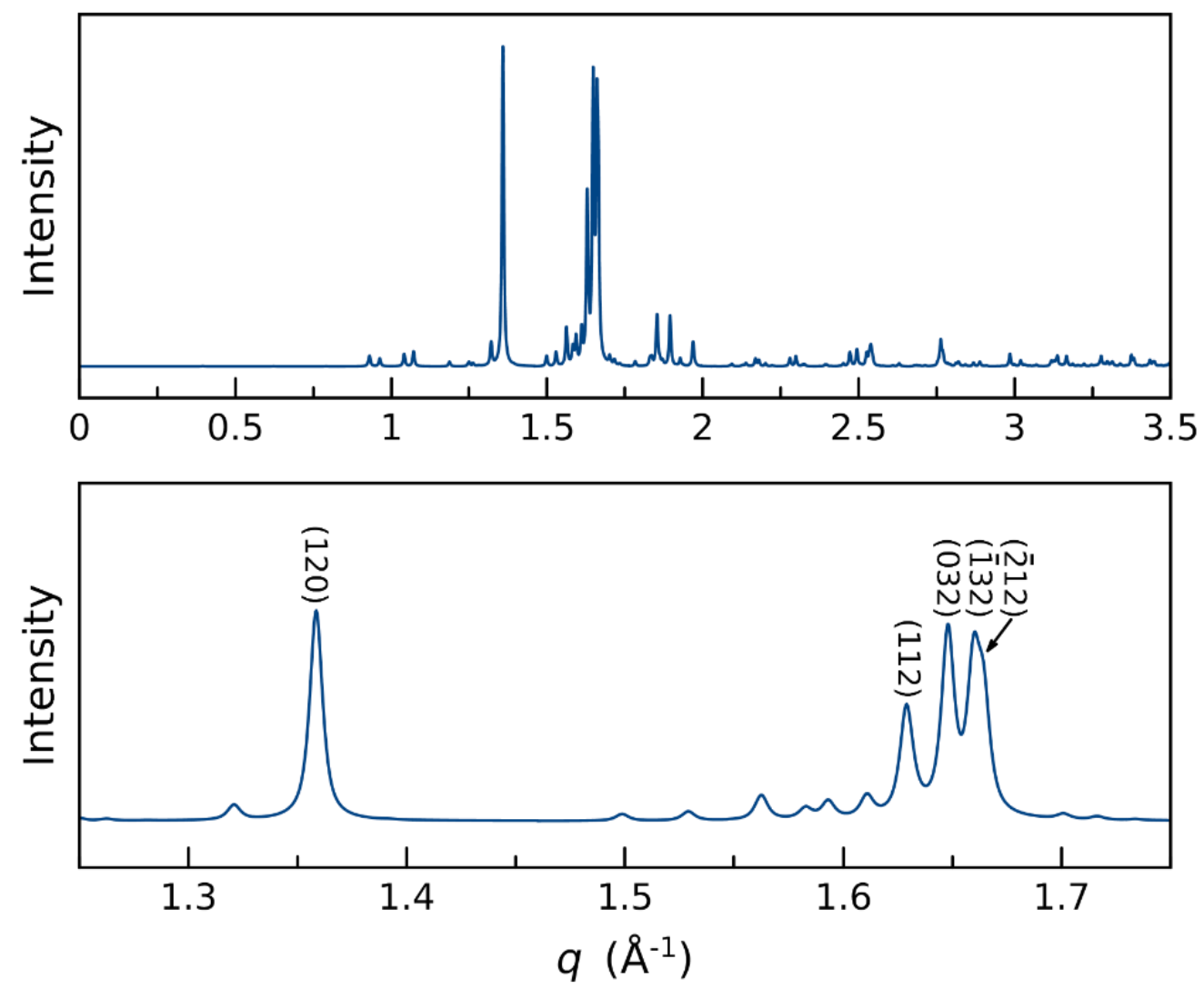

Figure S7: Calculated powder diffraction intensities of PEO as a function of $q$. Bottom figure is a zoomed-in view of the top figure. Calculations are based on the structural analysis by Takahashi et al. ${ }^{19}$

\begin{tabular}{cccccc}
\hline$H$ & $k$ & $l$ & $q\left(\AA^{-1}\right)$ & $d$-spacing $(\AA)$ & multiplicity \\
\hline 1 & 2 & 0 & 1.3585 & 4.625 & 4 \\
1 & 1 & 2 & 1.6289 & 3.8573 & 4 \\
0 & 3 & 2 & 1.648 & 3.8127 & 4 \\
-1 & 3 & 2 & 1.6598 & 3.7856 & 4 \\
-2 & 1 & 2 & 1.6644 & 3.7751 & 4
\end{tabular}

Table S1: Indexed $h k l$ reflection planes, $q, d$-spacing for the strongest PEO peaks, as shown in Figure S6. Indexing information of the weaker peaks be found in the Supplementary of Takahashi et al. ${ }^{19}$ 


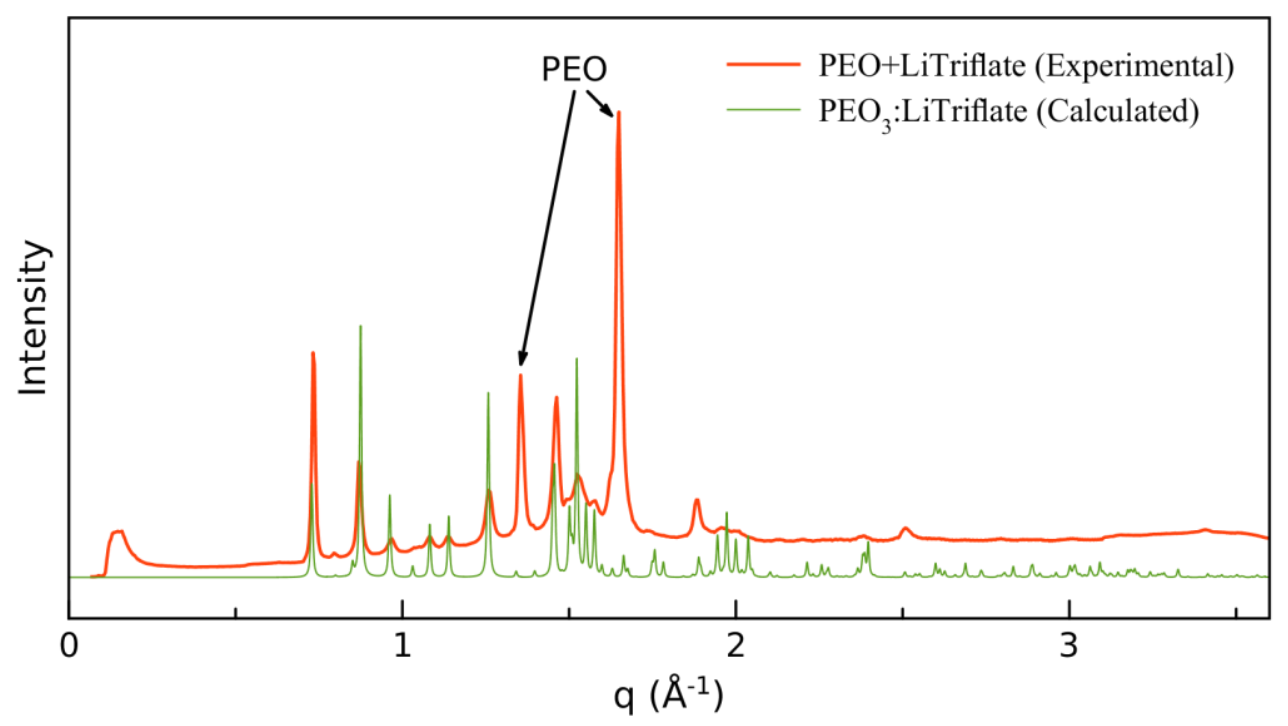

Figure S8: Calculated (green) and experimental (red) 1D intensities of PEO as a function of $q$. Calculations are based on the structural analysis of $\mathrm{PEO}_{3}$ :LiTriflate by Lightfoot et al. ${ }^{25}$ From the good match of peak positions, one can conclude that the PEO+LiTriflate sample measured experimentally exhibits a contribution from $\mathrm{PEO}_{3}$ :LiTriflate co-crystal domains.

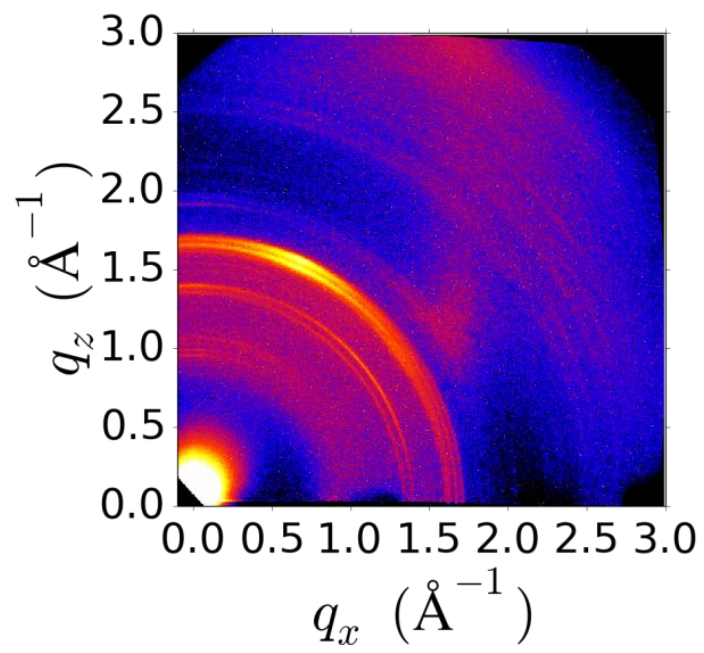

Figure S9: 2D GIWAXS pattern of PEO430 $k$ confined in $110 \mathrm{~nm}$ wide nanogratings, measured 16 months after thermal annealing. The same sample from the in-situ annealing experiment was used (green curve in Figure 5). The PEO crystallinity is stable at room temperature over extended periods. 


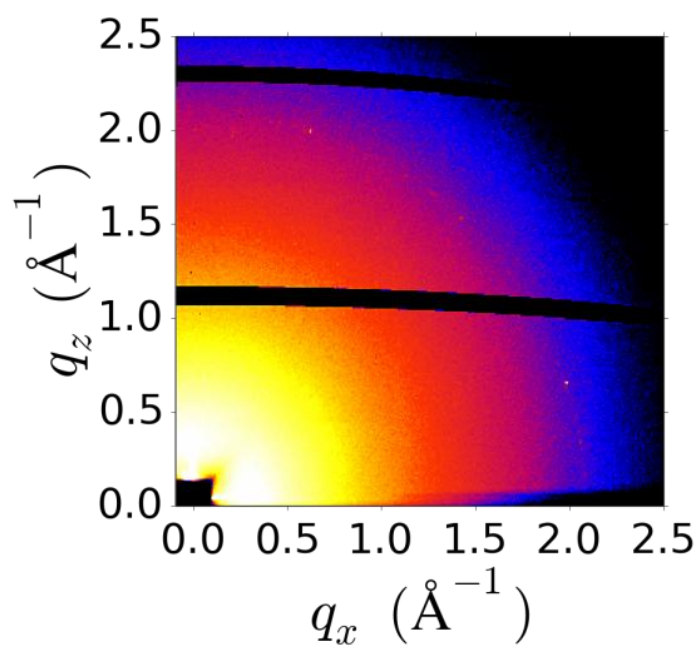

Figure S10: 2D GIWAXS pattern of PEO430 $k$ with LiTriflate salt confined in $110 \mathrm{~nm}$ wide nanogratings, measured 16 months after thermal annealing. The synergistic ability of confinement and salt to suppress PEO crystallization persists even after a long time period at room temperature. 